\title{
Algebraisch spezielle Einstein-Räume mit einer Bewegungsgruppe
}

\author{
D. Kramer und G. Neugebauer \\ Theoretisch-Physikalisches Institut \\ der Friedrich-Schiller-Universität Jena
}

Eingegangen am 15. September 1967

\begin{abstract}
In algebraically special Einstein spaces $\left(R_{\mu \nu}=0\right)$ with a hypersurfaceorthogonal spacelike Killing vector field $\xi_{v}$, the trajectories of the multiple eigen null directions $k_{\mu}$ lie - except one case - in the subspaces $V_{3}$ orthogonal to $\xi_{v}$ $\left(k_{\mu} \xi^{\mu}=0\right)$ and are hypersurface-orthogonal. The solutions with vanishing expansion $(k, \mu ; \mu=0$, Kundt's class $)$ can be determined explicitly.
\end{abstract}

In algebraisch speziellen Einsteinräumen $\left(R_{\mu \nu}=0\right)$ mit einem hyperflächennormalen raumartigen Killingvektorfeld $\xi_{v}$ liegen - mit Ausnahme eines Falles die Trajektorien der mehrfachen Null-Eigenrichtung $k_{\mu}$ in den $\mathrm{zu} \xi_{v}$ senkrechten Raumschnitten $\left(k_{\mu} \xi^{\mu}=0\right)$ und sind hyperflächennormal. Die Lösungen mit verschwindender Divergenz $\left(k_{, \mu} ; \mu=0\right.$, Kundtsche Klasse) können explizit bestimmt werden.

\section{Einleitung}

Das Ziel der Arbeit besteht in der möglichst expliziten Ermittlung aller entarteten Vakuumlösungen $\left(R_{\mu \nu}=0\right)$ mit einem hyperflächennormalen, raumartigen Killingvektor $\xi_{v}$.

Das Linienelement kann auf die Form ${ }^{1}$

$$
d s^{2}=V^{2}\left(d x^{3}\right)^{2}+g_{i j}\left(x^{k}\right) d x^{i} d x^{j}, \quad V=V\left(x^{i}\right), \quad \xi^{\mu}=\delta_{3}^{\mu}
$$

gebracht werden; es gelten dann die Reduktionsformeln

$$
\begin{aligned}
& \underset{(4)}{R_{3 i j k}}=0, \quad \underset{(4)}{R^{3}{ }_{i 3 j}}=-R_{i j}=\frac{V, i ; j}{V}, \quad R_{i}^{i}=0, \\
& \underset{(4)}{R_{i j k l}}=R_{i j k l}=-\varepsilon_{i j r} R^{r s} \varepsilon_{s k l} .
\end{aligned}
$$

Durch den Killingvektor $\xi_{\nu}$ sind Unterräume $V_{3}$ mit der Signatur $(-++)$ ausgezeichnet.

${ }^{1}$ Lateinische Indizes: $0 \ldots 2$; Griechische Indizes: $0 \ldots 3$.

12 Commun. math. Phys., Vol. 7 
Der reelle Nullvektor $k^{v}$ soll eine Eigenrichtung von $R_{\mu \nu \varrho \sigma}$ darstellen:

$$
k_{[\tau} R_{\nu] \varrho \sigma[\mu} k_{\lambda]} k^{\varrho} k^{\sigma}=0
$$

Das Kriterium für Entartung besagt:

$$
R_{\nu \varrho \sigma[\mu} k_{\lambda]} k^{\circ} k^{\sigma}=0
$$

Wir werden im weiteren zwei Fälle unterscheiden: $k^{\nu}$ hat entweder eine Komponente $k^{3} \neq 0\left(k_{v} \xi^{v} \neq 0\right)$ oder liegt ganz in $V_{3}\left(k_{v} \xi^{v}=0\right)$.

\section{Räume mit $\boldsymbol{k}_{\nu} \xi^{\nu} \neq 0$}

Mit (2) folgt aus den Kriterien (3), (4) unter der Voraussetzung $k_{\nu} \xi^{\nu} \neq 0$

Satz I. Liegt die isotrope Eigenrichtung $k_{v}$ nicht in dem zum Killingvektor senkrechten Unterraum $V_{3}\left(k_{v} \xi^{v} \neq 0\right)$, so sind die zugehörigen Räume vom Petrow-Typ I oder D. Typ I-Räume haben 0,2 oder 4 (einfache), Typ D-Räume 0 oder 2 (zweifache) isotrope Eigenrichtungen mit $k_{\nu} \xi^{\nu} \neq 0$.

Mehrfache Eigenrichtungen $k^{v}$ mit $k_{\nu} \xi^{v} \neq 0$ existieren genau dann, wenn $R_{i j}$ die Entwicklung

$$
R_{i j}=-\frac{V_{, i ; j}}{V}=\alpha\left(g_{i j}+3 u_{i} u_{j}\right), \quad u_{i} u^{i}=-1
$$

gestattet. Es gibt dann die beiden zweifachen Eigenrichtungen

$$
k_{v} \sim u_{v} \pm \frac{\xi_{v}}{\sqrt{\xi_{\mu} \xi^{\mu}}}, \quad u_{v}=\left(u_{i}, 0\right)
$$

Aus Geodäsie und Scherungsfreiheit von $k_{v}$ und unter Verwendung von (5) kann die Existenz eines hyperflächennormalen, zeitartigen Killingvektors $\eta_{v} \sim u_{v}$, gefolgert werden. Mit dieser Kenntnis lassen sich die Einsteinschen Feldgleichungen integrieren. Wir formulieren das Ergebnis in Klasse:

Satz II. Die einzige entartete Lösung mit $k_{v} \xi^{\nu} \neq 0$ gehört zur Weylschen

$$
d s^{2}=x^{1}\left(d x^{3}\right)^{2}+\left|\left(x^{1}\right)^{-1 / 2}\right|\left\{\left(d x^{1}\right)^{2}+\left(d x^{2}\right)^{2}\right\}-x^{1}\left(d x^{0}\right)^{2}, \quad x^{1}>0 .
$$

Diese Lösung ist in [1] invariant gekennzeichnet. 


\section{Räume mit $\boldsymbol{k}_{\nu} \xi^{\nu}=0$}

\subsection{Allgemeine Aussagen}

Das Kriterium (3) sowie die Bedingungen für mehrfache Entartung lassen sich auf kovariante Beziehungen in $V_{3}$ zurückführen:

$$
\begin{aligned}
& \text { Typ I: } & R_{i j} k^{i} k^{j} & =0 \\
& \text { Typ II: } & k^{i} R_{i[j} k_{m]} & =0 \\
& \text { Typ III: } & R_{i j} k^{j} & =0 \\
& \text { Typ N }: & R_{i[j} k_{m]} & =0 .
\end{aligned}
$$

Diese Klassifizierung steht in engem Zusammenhang mit den Eigenwerten und -vektoren von $R_{i j}$. Man erkennt aus (8.2), daß in allen entarteten Fällen eine Eigenwertgleichung

$$
R_{i j} k^{j}=\lambda k_{i}, \quad k_{i} k^{i}=0
$$

mit nullartigem Eigenvektor besteht, so daß sich Entartung in zusammenfallenden Eigenwerten von $R_{i j}$ äußert (vgl. z. B. [7]). $V_{4}$ ist also genau dann vom Typ I, wenn $R_{i j} 3$ verschiedene Eigenwerte $\lambda_{0}, \lambda_{1}, \lambda_{2}$ hat.

Um zu entscheiden, ob ein Typ I-Raum 4, 2 oder 0 Eigenrichtungen mit $k_{v} \xi^{\nu}=0$ (Satz I) hat, entwickeln wir $k_{i}$ nach dem Eigenvektorsystem von $R_{i j}$ und ermitteln die Zahl $n$ der verschiedenen reellen Lösungen $k_{i}$ von (8.1) in Abhängigkeit von den Eigenwerten:

$$
\begin{array}{ll}
3 \text { Eigenwerte reell, }\left(\frac{\lambda_{1}-\lambda_{2}}{\lambda_{0}}\right) \gtrless 9 & \begin{array}{l}
n=4^{2} \\
n=0
\end{array} \\
2 \text { Eigenwerte konjugiert-komplex } & n=2 .
\end{array}
$$

Bei sämtlichen Lösungen der Weylschen Klasse liegen entweder alle oder keine Eigenrichtungen $k_{v}$ vollständig in $V_{3}$.

Wir behandeln im folgenden die entarteten Lösungen mit $k_{\nu} \xi^{\nu}=0$. Mit Hilfe der Ricci-Identitäten und der Feldgleichungen folgt aus (9):

$$
\begin{gathered}
k^{j} k_{i ; j} \sim k_{i} \Leftrightarrow k_{i} \sim k_{, i} \\
\left(\frac{k^{i i}}{V}\right)_{; i}=0 .
\end{gathered}
$$

Diese Relationen drücken die Geodäsie und Scherungsfreiheit des Vektorfeldes $k_{v}=\left(k_{i}, 0\right)$ aus und sind auch aus den entsprechenden Aussagen in $V_{4}$ herleitbar. Wichtig ist in diesem Zusammenhang

Satz III. In $V_{3}$ ist für jeden Nullvektor $k_{i}$ Geodäsie gleichbedeutend mit Hyperflächennormalität.

$2 \lambda_{0}$ gehört zum zeitartigen Eigenvektor. 
Diese Aussage ergibt sich unmittelbar aus der Entwicklung von $k_{i ; j}$ nach einem Dreibein in $V_{3}$. Die Eigenschaft der Hyperflächennormalität überträgt sich natürlich auf den Vektor $k_{v}=\left(k_{i}, 0\right)$ in $V_{4}$. Daher gilt

Satz IV. Entartete Vakuumlösungen mit $k_{\nu} \xi^{\nu}=0$ gehören zu den von Kundt [3] $\left(k,{ }_{; \mu}=0\right)$ bzw. Robinson-Trautman [4] $\left(k^{, \mu}{ }_{; \mu} \neq 0\right)$ behandelten Lösungsklassen.

Die Voraussetzung einer Bewegungsgruppe gestattet in beiden Fällen explizitere Angaben über die Metrik.

Außer (11) gilt jetzt

$$
\text { 3.2. Kundtsche Klasse }\left(k^{, \mu}{ }_{; \mu}=0\right)
$$

und damit

$$
V k^{, \mu}{ }_{; \mu}=\left(V k^{, i}\right)_{; i}=0
$$

$$
V_{, i} k^{, i}=0 \text { : }
$$

Entweder ist $V_{, i}$ Nullvektor oder ein zu $k_{, i}$ orthogonaler raumartiger Vektor. Die Feldgleichungen haben nach einer Konformtransformation

die Gestalt

$$
g_{i j}=\frac{1}{V^{2}} \bar{g}_{i j}
$$

$$
\bar{R}_{i j}=-2 U_{, i} U_{, j}, \quad U=\log V .
$$

Im betrachteten Fall läßt sich unter teilweiser Verwendung der Feldgleichungen in einem für die Integration zweckmäßig gewählten Koordinatensystem $\left(k_{, i}=\delta_{i}^{0}, \bar{k}^{, i}=\delta_{1}^{i}\right)$ die Form des Linienelementes

$$
\begin{array}{rl}
\underset{(3)}{d \bar{s}^{2} \equiv \bar{g}_{i j} d x^{i} d x^{j}=} \bar{g}_{00}\left(d x^{0}\right)^{2}+2 & d x^{0} d x^{1}+ \\
& +\left(d x^{2}\right)^{2}+2 G\left(x^{0}, x^{2}\right) x^{1} d x^{2} d x^{0}, \\
\bar{g}_{00}=\frac{1}{2}\left(G^{2}-G_{, 2}\right)\left(x^{1}\right)^{2} & +E\left(x^{0}, x^{2}\right) x^{1}+F\left(x^{0}, x^{2}\right)
\end{array}
$$

erreichen. Die restlichen Feldgleichungen sind dem folgenden System von Differentialgleichungen äquivalent:

$$
\begin{aligned}
G_{, 2,2}-3 G,_{2} G+G^{3} & =0, \\
\left(U_{, 2}\right)^{2} & =\frac{1}{2}\left(G_{, 2}-\frac{1}{2} G^{2}\right), \\
E,_{2} & =G_{, 0}+4 U_{, 0} U_{, 2}, \\
\left(F_{, 2}+G F\right)_{, 2} & =-4\left(U_{, 0}\right)^{2} .
\end{aligned}
$$

$\mathrm{Zu}$ jeder Lösung der Differentialgleichung (16) kann man die Funktionen $U=U\left(x^{0}, x^{2}\right)$ und $E=E\left(x^{0}, x^{2}\right)$ aus (17) und (18) durch einfache 
Quadratur bestimmen; $F=F\left(x^{0}, x^{2}\right)$ ergibt sich aus der linearen Differentialgleichung (19). Die Feldgleichungen reduzieren sich also praktisch auf die Differentialgleichung (16), deren allgemeine Lösung angebbar ist:

$$
G=\frac{2\left(A-x^{2}\right)}{-B^{2}+\left(A-x^{2}\right)^{2}}, \quad A=A\left(x^{0}\right), \quad B=B\left(x^{0}\right) .
$$

Aus (17) erhält man nach einer noch ausführbaren Koordinatentransformation $\left(x^{0}=f\left(x^{0 \prime}\right), x^{1}=x^{1^{\prime}} \cdot\left(\dot{f}\left(x^{0 \prime}\right)\right)^{-1}, x^{2}=x^{2 \prime}\right)$

$$
U=\varepsilon x^{0}+\frac{1}{2} \log \left|\frac{x^{2}-A-B}{x^{2}-A+B}\right|, \quad \varepsilon=0, \pm 1 .
$$

Lösungen mit $B=0\left(U,{ }_{i} U, i=0\right)$ sind vom Petrow-Typ III, $N$ oder $O$. Die Differentialgleichung (16) hat die partikulären Lösungen

$$
\begin{gathered}
G=\frac{1}{A-x^{2}}, \\
G=0 \quad(\operatorname{Typ} N, O) .
\end{gathered}
$$

Ergebnis dieses Abschnittes ist

Satz V. Alle Lösungen der Kundtschen Klasse $\left(k^{, \mu}{ }_{; \mu}=0\right)$ mit (mindestens) einem hyperflächennormalen Killingvektor $\xi_{\mu}$ sind durch (15)-(22) explizit bestimmt.

\subsection{Robinson-Trautmansche Klasse $\left(k^{, \mu} ; \mu \neq 0\right)$}

In diesem Fall lösen wir die Killinggleichung

$$
\xi_{\mu ; \nu}+\xi_{v ; \mu}=0
$$

in der von Robinson-Trautuan [4] angegebenen Normalform der Metrik:

$$
d s^{2}=\frac{\varrho^{2}}{p^{2}}\left(d \xi^{2}+d \eta^{2}\right)-2 d \varrho d x^{0}-L\left(d x^{0}\right)^{2}, \quad \frac{\partial p}{\partial \varrho}=0 .
$$

Dabei ist:

$$
\begin{aligned}
& L=-\frac{2 m}{\varrho}+K-2 H \varrho, \quad m=m\left(x^{0}\right), \\
& H=\frac{\partial \log p}{\partial x^{0}}, \quad K=p^{2}\left(\frac{\partial^{2}}{\partial \xi^{2}}+\frac{\partial^{2}}{\partial \eta^{2}}\right) \log p .
\end{aligned}
$$

Mit (24), (25) sowie mit $k_{\nu} \xi^{\nu}=0$ erhalten wir aus (23) die Aussagen:

$$
\begin{gathered}
\xi^{\mu}=\left(\xi^{1}, \xi^{2}, 0,0\right) \\
\xi^{1}+i \xi^{2}=f(z), z=x^{1}+i x^{2}\left(x^{1} \equiv \xi, x^{2} \equiv \eta\right) f(z) \text { analytisch }, \\
\left(\frac{\xi^{A}}{p^{2}}\right)_{, A}=0, \quad A=1,2 .
\end{gathered}
$$


Der Killingvektor erweist sich wegen (26) automatisch als hyperflächennormal. Durch eine Transformation $f(z) \rightarrow z$, bei der die Form des Linienelementes (24) stets erhalten bleibt, erreicht man wegen (27) $\xi^{1}=x^{1}$, $\xi^{2}=x^{2}$; und die allgemeine Lösung von (28) lautet dann in diesen Koordinaten ${ }^{3}$

$$
p=x^{1} \cdot Q\left(\frac{x^{1}}{x^{2}}, x^{0}\right) .
$$

Den anschließenden Übergang zu einem Kugelflächenelement im ZweierUnterraum vermittelt die Transformation

$$
\begin{aligned}
& x^{1}=e^{\varphi} \cdot \sin \left(\log \operatorname{tg} \frac{\vartheta}{2}\right) \\
& x^{2}=e^{\varphi} \cdot \cos \left(\log \operatorname{tg} \frac{\vartheta}{2}\right) .
\end{aligned}
$$

Die Metrik erhält in Übereinstimmung mit $(1)\left(x^{3} \equiv \varphi\right)$ die Form

$$
\begin{gathered}
d s^{2}=q^{2}\left(\vartheta, x^{0}\right) \varrho^{2}\left(\sin ^{2} \vartheta d \varphi^{2}+d \vartheta^{2}\right)-2 d \varrho d x^{0}-L\left(d x^{0}\right)^{2} \\
L=\frac{-2 m}{\varrho}+K-2 H \varrho, \quad H=-\frac{\partial \log q}{\partial x^{0}}, \quad m=m\left(x^{0}\right) .
\end{gathered}
$$

Die Feldgleichungen fordern schließlich ${ }^{4}$

$$
\begin{aligned}
K & =\frac{-1}{q^{2} \sin \vartheta}\left(\frac{(q \sin \vartheta)^{\prime}}{q}\right)^{\prime} \\
\frac{1}{12} \frac{\left(K^{\prime} \sin \vartheta\right)^{\prime}}{q^{2} \sin \vartheta} & =m \frac{\partial \log q}{\partial x^{0}}, \quad m=\text { const . }
\end{aligned}
$$

Es gilt somit

Satz VI. Alle Lösungen der Robinson-Trautmanschen Lösungsklasse $\left(k^{, \mu}{ }_{; \mu} \neq 0\right)$ mit axialer Symmetrie sind aus (32) bestimmbar.

Es ist uns nicht gelungen, die allgemeine Lösung des Differentialgleichungssystems (32) zu finden. Für $m=0$ ergibt sich

$$
K=A\left(x^{0}\right) \cdot \log \operatorname{tg} \frac{\vartheta}{2}+B\left(x^{0}\right) .
$$

Hier liegt eine Singularität längs der Achse $\vartheta=0, \pi$ vor. Aus der Form des Linienelementes (31) erkennt man: Lösungen, die für $\varrho \rightarrow \infty$ pseudoeuklidische Form annehmen, sind durch $q^{2}=1$ gekennzeichnet; denn $q$ ist $\varrho$-unabhängig. Die einzige Lösung, die dieser Bedingung genügt, ist die Schwarzschild-Lösung $\left(q^{2}=K=1\right)$.

\subsection{Geodätische Eigenrichtungen}

Eine Verschärfung unserer letzten Feststellung bildet

Satz VII. Außer der Schwarzschildlösung gibt es keine axialsymmetrische Lösung der Einsteinschen Feldgleichungen, die das Feld einer

${ }^{3} Q$ : beliebige Funktion der angegebenen Argumente.

${ }^{4}$ Strich bedeutet Ableitung nach $\vartheta$. 
isolierten Quellverteilung beschreibt und gleichzeitig eine geodätische Eigenrichtung von $R_{\mu \nu \rho \sigma}$ mit $k_{\nu} \xi^{\nu}=0$ besitzt.

Den Beweis führen wir in einem Bondischen Koordinatensystem, in dem sich alle realistischen Gravitationsfelder in größerer Entfernung von der isolierten Quellverteilung beschreiben lassen. Wir verwenden die geodätische Eigenkongruenz $k^{\mu}$ zum Aufbau der Bondi-Metrik [5]:

$$
\begin{gathered}
k^{\mu}=\left(0, k^{1}, 0,0\right), \quad \xi^{\mu}=\left(0,0,0, \xi^{3}\right) \\
d s^{2}=\varrho^{2}\left(e^{-2 \gamma} \sin ^{2} \vartheta d \varphi^{2}+e^{2 \gamma} d \vartheta^{2}\right)-2 U \varrho^{2} e^{2 \gamma} d x^{0} d \vartheta- \\
-2 e^{2 \beta} d x^{0} d \varrho+g_{00}\left(d x^{0}\right)^{2} . \quad\left(x^{1} \equiv \varrho, x^{2} \equiv \vartheta, x^{3} \equiv \varphi\right) .
\end{gathered}
$$

Die Bedingung, daß $k^{\mu}$ Eigenrichtung des Riemanntensors ist, lautet nach (8.1)

$$
V_{, 1 ; 1}=0, \quad V=\varrho e^{-\gamma} \sin \vartheta .
$$

Im Zusammenhang mit der Feldgleichung [5]

$$
\beta_{, 1}=\frac{1}{2} \varrho(\gamma, 1)^{2}
$$

erhält man die Lösung

$$
\gamma=\mathfrak{A r} \operatorname{Sin} \frac{c}{\varrho}, \quad \beta=-\frac{1}{4} \log \left(1+\frac{c^{2}}{\varrho^{2}}\right), \quad c=c\left(x^{0}, \vartheta\right),
$$

wobei eine Integrationsfunktion bereits so gewählt wurde, daß $\beta$ und $\gamma$ für $\varrho \rightarrow \infty$ das richtige asymptotische Verhalten haben. Die Funktion $\gamma$ hat die allgemeine Entwicklung

$$
\gamma=c \varrho^{-1}+\left[C-\frac{1}{6} c^{3}\right] \varrho^{-3}+D \varrho^{-4}+\cdots
$$

Bei dem gefundenen Ausdruck (37) für $\gamma$ ist also $C=D=0$. Damit ergeben sich aus den Feldgleichungen unter anderem die zusätzlichen Forderungen (vgl. auch [6])

Aus (39) folgt

$$
\begin{gathered}
(c N)_{, 2}+3 c N \cot \vartheta=0 \\
2 c^{2} c,_{, 0}+2 c M+N \cot \vartheta-N_{, 2}=0 .
\end{gathered}
$$

$$
c N=\frac{h\left(x^{0}\right)}{\sin ^{3} \vartheta} .
$$

Für $h\left(x^{0}\right) \neq 0$ ist dies nicht mit den Regularitätsbedingungen auf der Achse $\vartheta=0, \pi$ vereinbar, die zumindest in großem Abstand von der isolierten Quelle erfüllt sein müssen. Für $h\left(x^{0}\right)=0$ ergibt sich als einzige reguläre Metrik die Schwarzschildlösung $(c=0)$.

Herrn Prof. Dr. E. Schmutzer und den Mitgliedern der Arbeitsgruppe Relativitätstheorie danken wir für wertvolle Diskussionen. 


\section{Literatur}

1. Jordan, P., J. Ehlers u. W. Kundt: Strenge Lösungen der Feldgleichungen der Allg. Relativitätstheorie; Abh. Mainz. Akad. math.-naturw. Kl. 1960, Nr. 2, S. 74.

2. ebenda, S. 83.

3. KUndt, W., Z. Physik, 163, 77 (1961).

4. Robinson, I., and A. Trautman, Proc. Roy. Soc. A, 265, 463 (1962).

5. Bondi, H., M. G. J. van der Burg, and A. W. K. Metzner, Proc. Roy. Soc. A, 269, 21 (1962).

6. van der Burg, M. G. J., Proc. Roy. Soc. A 294, 112 (1966).

7. Landau-Lifschitz - Lehrbuch der Theoret. Physik, Band II, S. 385. Berlin: Akad. Verlag 1963. 